\title{
Angina Instável devida a Comunicação entre Artéria Coronária e Artéria Pulmonar Direita em Paciente com Arterite de Takayasu
}

\author{
Paulo R. Viecili, David Pamplona, Fernando H. Y. Cesena, Protásio L. da Luz
}

São Paulo, SP

Mulher de 63 anos com arterite de Takayasu, envolvendo arco aórtico, artérias subclávias e artéria pulmonar direita, com dor precordial progressiva. $O$ eletrocardiograma de esforço revelou depressão do segmento ST. A cineangiocoronariografia não mostrou lesões estenóticas ostiais e nem epicárdicas e, sim, comunicação entre a artéria coronária circunflexa e o ramo do lobo superior da artéria pulmonar direita. O fluxo sangüíneo através da comunicação arterial foi considerado de grau importante, provocando, provavelmente, síndrome do roubo de fluxo coronário e, conseqüentemente, o quadro de angina de peito com caráter progressivo.

\section{Coronary Steal due to Collateral Circulation from Circumflex Coronary to Right Pulmonary Artery Leading to Unstable Angina in Takayasu's Arteritis}

A sixty three year old woman suffering from Takayasu's arteritis with involvement of the aortic arch, subclavia arteries and occlusion of the right pulmonary artery, developed progressive precordial pain. Exercise electrocardiogram disclosed ST segment depression. Coronary arteriograms demonstrated no coronary stenosis, but collateral circulation arising from circumflex coronary artery to right pulmonary artery. The collateral blood flow was considered to be of great importance, and causing the coronary steal syndrome, leading to angina pectoris.

Arq Bras Cardiol, volume 69 (nº 2), 129-132, 1997

A arterite de Takayasu (AT), ao longo de sua evolução, produz danos severos ao sistema cardiocirculatório ${ }^{1-3}$, sendo a isquemia miocárdica uma das mais letais ${ }^{4}$. Apesar de esporádica, tem-se descrito como secundária a lesões coronárias ostiais ou epicárdicas proximais, em decorrência do prolongamento do processo inflamatório ${ }^{4}$. Mais recentemente, tem-se relatado a ocorrência de fístulas, circulação colateral e comunicações arteriais complicando a $\mathrm{AT}^{4-6}$.

Nosso objetivo é relatar um caso de forma muito rara de isquemia miocárdica, diferente dos já apresentados.

\section{Relato do Caso}

Mulher de 63 anos de idade, branca, com antecedente de AT há 20 anos, foi admitida, em serviço de emergência,

Instituto do Coração do Hospital das Clínicas - FMUSP

Correspondência: Paulo Ricardo Viecili - Incor - Unidade de Emergência - Av. Dr. Enéas C. Aguiar, 44 - 05403-000 - São Paulo, SP

Recebido para publicação em 23/1/97

Aceito em 11/6/97 com queixa de desconforto precordial aos míninos esforços e com episódios de angina, iniciando-se aos médios esforços, há seis meses, e progredindo aos mínimos esforços, nas últimas semanas.

Ao exame físico, apresentava-se em bom estado geral, eupnéica, acianótica, os pulsos não eram palpáveis em membros superiores e os dos membros inferiores tinham amplitudes normais e eram simétricos. A frequiência cardíaca era de $80 \mathrm{bpm}$ e a pressão arterial de $170 \times 80 \mathrm{mmHg}$, aferida nos membros inferiores. Não apresentava sinais de insuficiência cardíaca e nem outros comemorativos.

O eletrocardiograma (ECG), realizado na admissão hospitalar, revelou alterações difusas da repolarização ventricular e a radiografia de tórax não apresentava alterações significativas. Vinha acompanhada de exames realizados previamente, sendo os mais importantes: ECG de esforço (fig. 1) com alterações importantes da repolarização ventricular, principalmente em paredes inferior e lateral; ecocardiograma com discreto aumento do diâmetro da raiz da aorta, câmaras cardíacas de volumes e mobilidade normais, espessura miocárdica do ventrículo direito (VD) e es- 


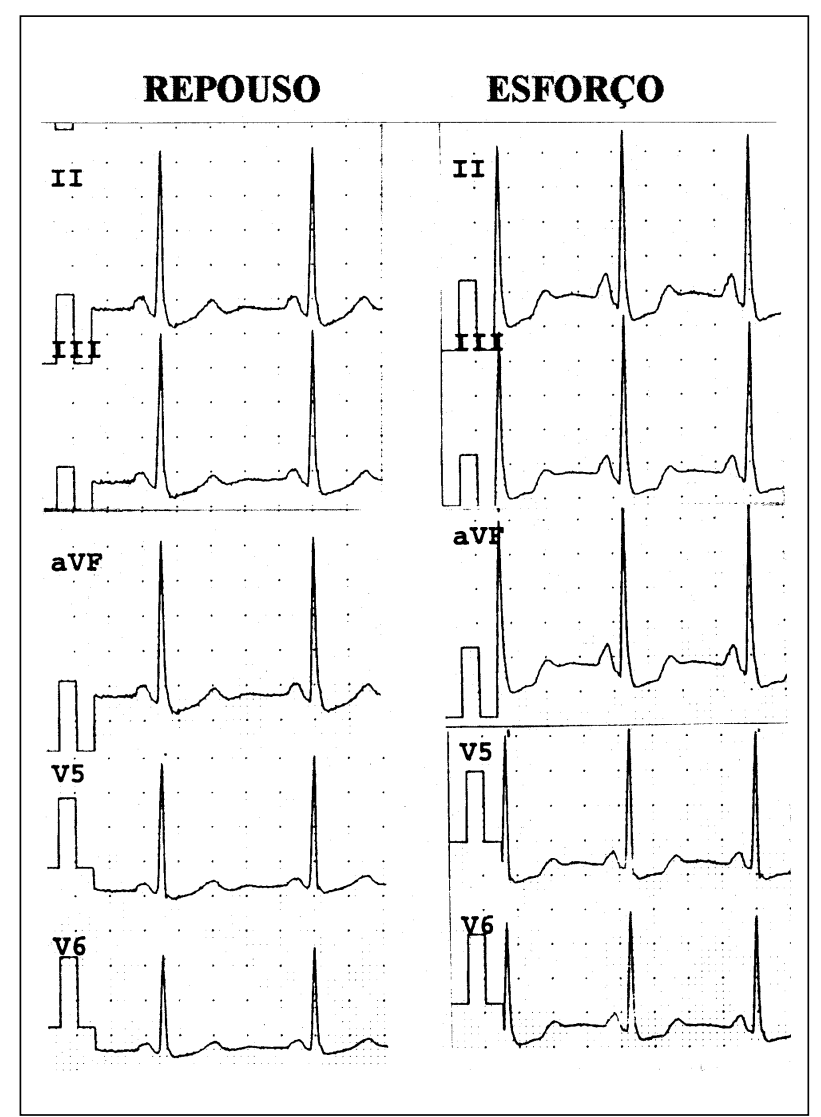

Fig. 1 - Teste de esforço: derivações DII, DIII, aVFe $\mathrm{V}_{5}-\mathrm{V}_{6}$, correspondendo às paredes inferior e lateral, respectivamente. Observe a nítida modificação na morfologia do segmento ST do repouso para o exercício. querdo (VE) normais e valvas sem alterações anatômicas; arteriografia de arco aórtico e troncos braquiocefálicos com oclusão de ambas as artérias subclávias em suas origens, com extensa rede de colaterais para as mesmas e alterações discretas do arco aórtico.

Internada com diagnóstico de angina progressiva e medicada com nitrato via oral, inibidores de canais de cálcio e ácido acetil salicílico permaneceu assintomática.

$\mathrm{Na}$ arteriografia coronária (fig. 2) não havia lesões obstrutivas nas artérias coronárias, no entanto, a artéria do lobo superior direito contrastava-se a partir da artéria circunflexa. A manometria em repouso revelava hipertensão pulmonar com 60/20mmHg, VEcom 180/15mmHg e aorta com 180/90mmHg. Na aortografia (fig. 3), observaram-se irregularidades no arco aórtico e obstrução das artérias subclávias. A arteriografia pulmonar mostrou amputação da artéria do lobo superior direito.

Recebeu alta com medicação e foi orientada quanto à limitação de suas atividades físicas.

\section{Discussão}

A AT é uma arterite primária, provavelmente autoimune, que comumente afeta a aorta torácica e seus ramos principais e aorta abdominal ${ }^{7-9}$. Embora seja encontrada com maior frequiência na Índia e Japão, tem distribuição universal, com prevalência maior em mulheres jovens ${ }^{10,11}$.

Em sua apresentação clássica, a doença manifesta-se basicamente de duas formas: $1^{a}$, mais precoce, caracteriza-

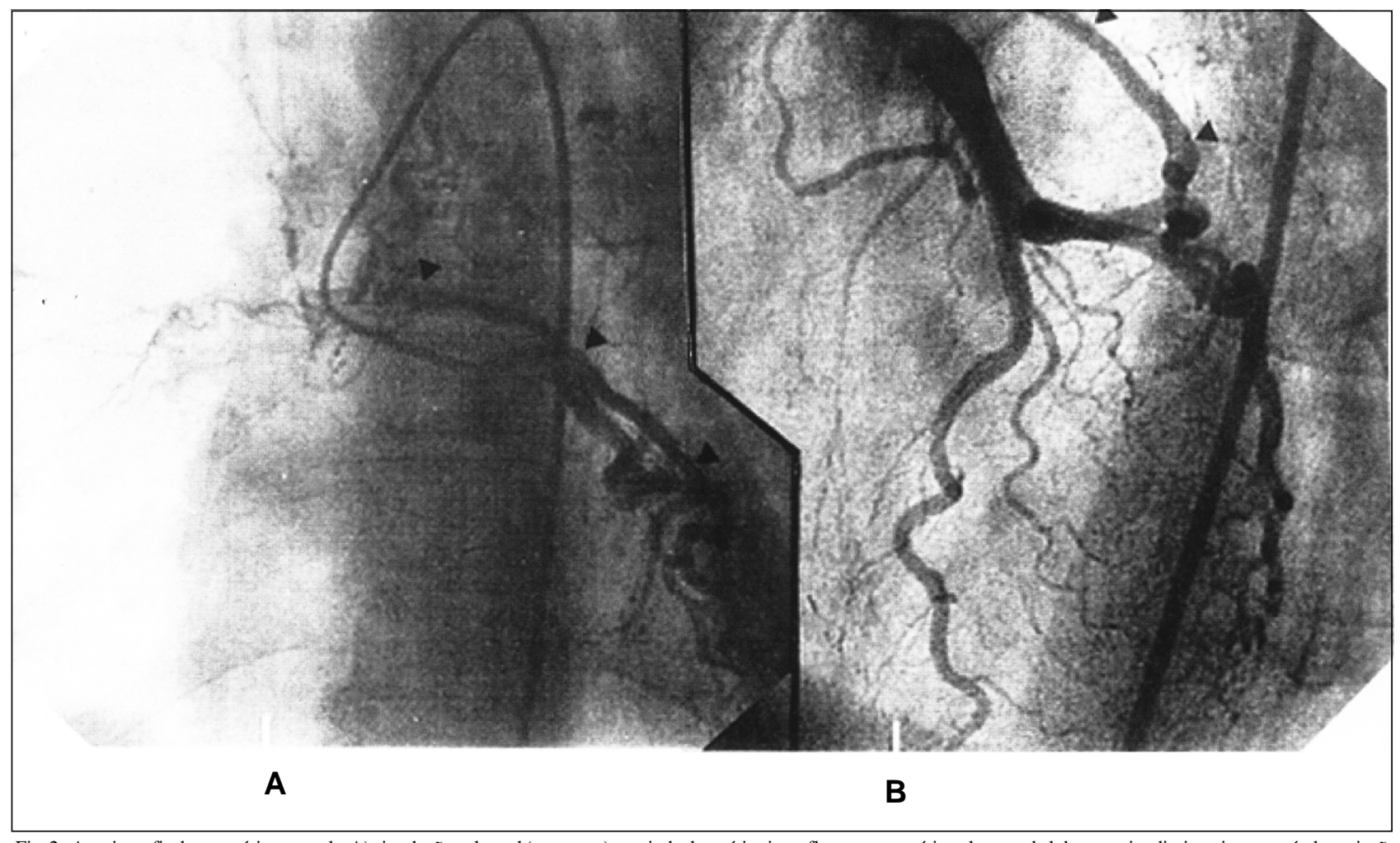

Fig. 2 - Arteriografia da coronária esquerda. A) circulação colateral (seta preta), partindo da artéria circunflexa para a artéria pulmonar do lobo superior direito, vista através da projeção oblíqua anterior direita; B) porção inicial da circulação colateral (seta preta), partindo da artéria circunflexa, vista através da projeção oblíqua anterior esquerda. 
se por sintomas gerais e inespecíficos, como febre, mal estar, perda ponderal e artralgias; a $2^{\mathrm{a}}$, mais tardia, caracteriza-se por quadro clínico de obstrução arterial, com sinais e sintomas dependendo da intensidade do acometimento no sistema cardiocirculatório, incluindo as artérias pulmonares ${ }^{10}$.

Suas principais complicações envolvendo o sistema cardiocirculatório são: hipertensão arterial sistêmica em 58 a $88 \%$ dos casos ${ }^{10-14}$; insuficiência coronária (Ico) entre 9 a $25 \%{ }^{15,16}$ e insuficiência cardíaca congestiva, $8 \%$ em adultos e $70 \%$ em crianças ${ }^{15,17}$.

A evolução para Ico pode ser atribuída a mecanismos como: 1) estreitamento ostial coronário por causa do prolongamento do processo inflamatório arterial, não havendo predileção coronária e podendo acometer mais de uma coronária; 2) por lesão coronária epicárdica aterosclerótica não relacionada a AT; 3) pelas duas formas anteriores associadas ${ }^{10,15}$;) por lesão da microcirculação coronária ${ }^{10,18} ; 5$ ) por hipertrofia miocárdica; 6) insuficiência aórtica ${ }^{19}$; e por último, pela rara presença de comunicações arteriais envolvendo as artérias coronárias ${ }^{2,20}$.

Existem relatos de comunicações arteriais da coronária direita, através do comprometimento da artéria do nó sinusal, com as artérias vertebrais e a artéria pulmonar direita ${ }^{10-11}$, assim como da artéria do nó sinusal, com origem da artéria circunflexa, para a artéria brônquica esquerda ${ }^{4}$. As comunicações arteriais na AT, primeiramente descritas por Ishikawa ${ }^{18}$, podem não só envolver as artérias coronárias, como principalmente as artérias brônquicas, artérias pulmonares, mamária interna, torácica lateral, pericardiofrênica, intercostais e inominata ou através de reflexão mediastinal, ligamento pulmonar e adesões pleurais ${ }^{19-22}$.

Em nosso caso, o diagnóstico de AT confirmou-se pela história prévia e pelos achados angiográficos, como o comprometimento do arco aórtico, oclusão das artérias subclávias e da artéria do lobo superior direito. A incidência do acometimento das artérias pulmonares aproxima-se de $14 \%$ na AT e destas $66 \%$ estão localizadas no lobo superior direito ${ }^{22}$. Por outro lado, existe uma forte correlação entre o grau de comprometimento das artérias pulmonares e a presença de comunicações arteriais ${ }^{7}$ como encontrado nesta paciente. A evolução para angina instável, sem apresentar lesões ostiais e/ou ateroscleróticas, insuficiência aórtica e nem hipertrofia miocárdica, foi atribuído à síndrome do roubo do fluxo sangüíneo da coronária circunflexa para a artéria pulmonar.

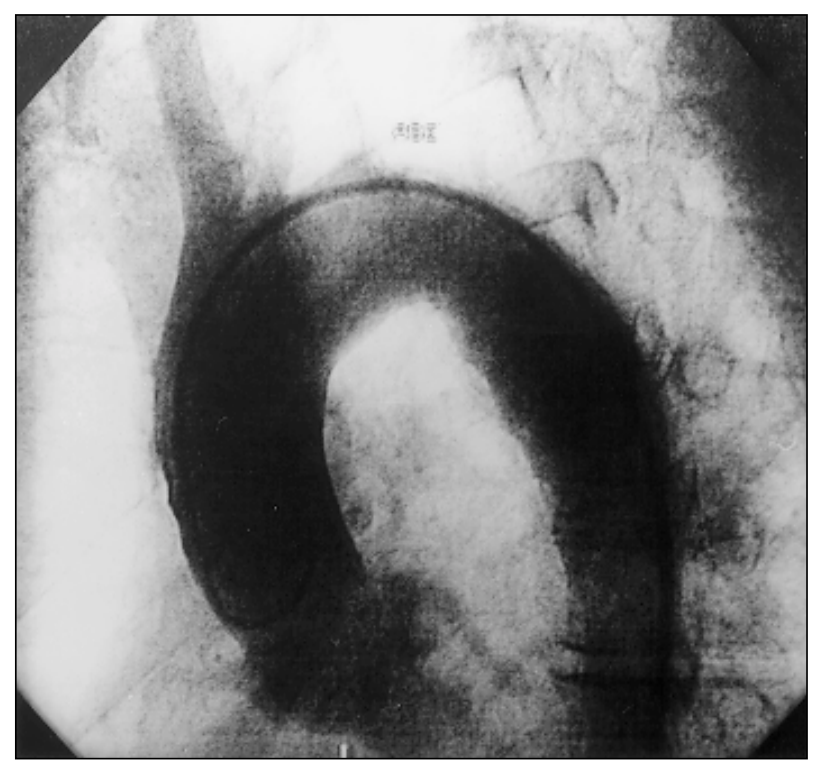

Fig. 3 - Aortografia: observar a oclusão de ambas as artérias subclávias.

Moberg ${ }^{23}$ sugere que a presença de comunicação arterial na AT exista desde a época do nascimento, porém de tamanho e importância hemodinâmica insignificantes. As comunicações podem se tornar funcionais quando um gradiente de pressão significativo existir entre os vasos envolvidos ${ }^{23}$. Já foi demonstrado, em pacientes com doença aterosclerótica coronária e AT, que o fluxo coronário pode se originar da artéria brônquica, quando nesses territórios este fluxo estiver reduzido. Por outro lado, se houver diminuição da perfusão do leito vascular pulmonar, pelo acometimento das artérias brônquicas ou pulmonares, pode haver reversão da direção do fluxo sangüíneo ${ }^{24}$. A falta de lesões ostiais ou ateroscleróticas nas coronárias e o comprometimento da vasculatura pulmonar contribui para essa hipótese.

Nossa paciente foi tratada com fármacos vasodilatadores e limitação física, não sendo proposta a embolização arterial nem a correção cirúrgica, preconizadas por alguns autores ${ }^{25}$, devido à grande área pulmonar comprometida.

Concluimos deste relato que, além das causas clássicas esperadas de angina instável na AT, tem que se ter em mente, principalmente quando a circulação pulmonar estiver comprometida, a possibilidade da existência de comunicações arteriais, fístulas ou circulações colaterais, aumentando a complexidade dessa doença.

\section{Referências}

1. Makino N, Orita Y, Takeshita A, Motomi N, Mastsui K, Tokunaga K - Coronary arterial involvement in Takayasu's disease. Jpn Heart J 1982; 23: 1007-13.

2. Nasu T - Autopsy study of aortitis syndrome. Report by the Ministry of Health and Welfare. Japan, 1975: 111.

3. Huang PJ, Chu SH, Chen CM - Takayasu's arteritis with coronary occlusion and reinfarction angina. Formosa Med Assoc 1975; 74: 469.

4. Lupi EH, Sánchez GT, Howitz S, Gutierrez EF - Pulmorary artery involvement in Takayasu's arteritis. Chest 1975; 67: 69-74.

5. Masugata H, Yasuno M, Nishino Met al - Takayasu's arteritis with collateral circulation from the right coronary artery to intracranial vessels. A case report. Angiology-J Vasc Dis 1992; 5: 448-52.
6. Sumitani T, Genda A, Kanayama Het al - A case of aortitis syndrome with the collateral circulation from the coronary artery and the right bronchial artery to the lung. Kokyu to Junkan 1982; 30: 1167-72.

7. Yamada I, Shibuya H, Matsubara O et al - Pulmonary artery disease in Takayasu's arteritis: Angioraphic findings. AJR 1992; 159: 263-9.

8. Grollman JH Jr, Hanafee W - The roentgen diagnosis of Takayasu's arteritis. Radiology 1963; 83: 387-95.

9. Lande A, Gross A - Total aortograhy in the diagnosis of Takayasu's arteritis. AJR 1972; 116: 165-78.

10. Manotosh P, Kar AK, Dutta AL, Chetri M, Kumar S, Panja S - Cardiac involvement in non-specific aorto-arteritis. Intern J Cardiol 1992; 34: 289-95. 
11. Horimoto M, Igarashi K, Okamoto K, Takenaka T - Unilateral diffuse pulmonary artery involvement in Takayasu's arteritis associated with coronary-pulmonary artery fistula and bronchial-pulmonary artery fistula. Angiology-J Vasc Dis 1991; 42:73-80.

12. Nakao K, Ideda M, Kimata S et al - Takayasu's arteritis: clinical report of 85 cases and immunological study of seven cases. Circulation 1967; 35: 1141-55.

13. Das JP, Misra H, Dasit MA, Nayak Cr, Swain U - Nom-specific arteritis. Indian Heart J 1982; 34: 391-4.

14. Majumdar S, Mulheerjee R, PaulDK, Batabyal SK, Hundu SC - Aortoarteritis in Eastern India - a 15-year study. Indian Heart J 1982; 34: 281-5.

15. Kinare SG - Cardiac lesions in non-specific aorto-arteritis an autopsy study. Indian Heart J 1994; 46: 65-9.

16. Rose AG, Sinclair-Smith CC - Takayasu's arteritis - A study of 16 autopsy cases. Arch Pathol Lab Med 1980; 104: 231-4.

17. Talwar KK, Chopra P et al - Cardiac involvement in non-specific aorto-arteritis. Am Heart J 1992; 122: 1660-6.

18. Ishikawa $\mathrm{K}$ - Diagnostic approach and proposed critera for the clinical diagnosis of Takayasu's arteriopathy. J Am Coll Cardiol 1988; 12: 964-7.
19. Lupi-HerreraE, Sanchez-Torres G, Marcushamer J, Mispireta J, HorwitzS, Vela JE - Takayasu's arteritis. Clinical study of 107 cases. Am Heart J 1977; 93: 94-8.

20. Ishikawa $\mathrm{T}$ - Systemic artery-pulmonary artery comunication in Takayasu's arteritis. Am J Roentgenol 1977; 128: 389-97.

21. Massumi RA, Rios JC, Donohoe RF - The pathogenesis of angiographic nonvisualization or attenuation of a patent pulmonary artery and the role of bronchial-pulmonary artery anstomosis. J Thorac Cardiovasc Surg 1965; 49: 772-89.

22. Sharma S, Kamalakar T, Rajani M, Talwar K, Shrivastava S - The incidence and patterns of pulmonary artery involvement in Takayasu's arteritis. Clin Radiol 1990; 42: 177-81

23. Moberg A - Anastomoses between extracardiac vessels and coronary arteries. Acta Med Scand 1968; (suppl 485): 5-26.

24. Smith SC, Adams DF, Herman MU, Paulin S - Coronary-to-bronchial anastomosis: an in vivi demonstration by seletive coronary arteriography. Radiology 1972; 104: 289-90.

25. Lopez AJ, Brady JB, Jackson JE- Case Report: Therapeutic bronchial artery embolization in a case of Takayasu's arteritis. Clin Radiol 1992; 45: 415-17. 